Research Article

\title{
Reinforcement Number of a Graph with respect to Half-Domination
}

\author{
G. Muhiuddin $\mathbb{D}^{1},{ }^{1}$ N. Sridharan, ${ }^{2}$ D. Al-Kadi, ${ }^{3}$ S. Amutha $\left(\mathbb{D},{ }^{4}\right.$ and M. E. Elnair ${ }^{1,5}$ \\ ${ }^{1}$ Department of Mathematics, University of Tabuk, Tabuk 71491, Saudi Arabia \\ ${ }^{2}$ Department of Mathematics, Alagappa University, Karaikudi 630 003, India \\ ${ }^{3}$ Department of Mathematics and Statistics, College of Science, Taif University, P.O. Box 11099, Taif 21944, Saudi Arabia \\ ${ }^{4}$ Ramanujan Centre for Higher Mathematics, Alagappa University, Karaikudi 630 003, India \\ ${ }^{5}$ Department of Mathematics and Physics, Faculty of Education, Hasahesa, Gezira University, Sudan
}

Correspondence should be addressed to S. Amutha; amuthas@alagappauniversity.ac.in

Received 17 December 2020; Revised 1 February 2021; Accepted 30 March 2021; Published 14 April 2021

Academic Editor: Ismail Naci Cangul

Copyright (C) 2021 G. Muhiuddin et al. This is an open access article distributed under the Creative Commons Attribution License, which permits unrestricted use, distribution, and reproduction in any medium, provided the original work is properly cited.

In this paper, we introduce the concept of reinforcement number with respect to half-domination and initiate a study on this parameter. Furthermore, we obtain various upper bounds for this parameter. AMS subject classification: 05C38, 05C40, 05 C69.

\section{Introduction}

Throughout our discussion, we consider only simple finite graphs. For graph theoretic terminologies, we refer the readers to [1]. If $G=(V, E)$ is a graph to each vertex $v$ of $G$, $N_{1}(v)$ denotes the set of all vertices of $G$ which are adjacent to $v$. For any subset $S \subseteq V, N_{1}(S)=\cup\left\{N_{1}(v): v \in S\right\}$. For a vertex $v$ of $G, N_{1}[v]=N_{1}(v) \cup\{v\}$ and $N_{2}[v]=\{u \in V: \mathrm{d}$ $(u, v)=2\}$. A vertex $v \in V$ is said to dominate itself and its adjacent vertices. In other words, a vertex $v$ dominates a vertex $u$ if $u \in N_{1}[v]$. A subset $D$ of $V(G)$ is said to be a dominating set of $G$ if $V=\cup_{v \in D} N_{1}[v]$. The minimum cardinality of a dominating set $D$ of $G$ is denoted by $\gamma(G)$ and is called the domination number of $G$. The total domination number of a graph $\mathrm{G}$, denoted by $\gamma_{t}(G)$, is the minimum cardinality of a total dominating set of $G$ and, for their properties, we refer the reader to [2-6]. In [7], Kulli introduced the concept of the cobondage number $c b(G)$ of a graph, which is the minimum number of edges to be added to reduce the domination number. The same concept has been independently introduced and studied earlier by others under the name "reinforcement number" (refer to chapter 17 of Haynes et al.'s work [8]). Total reinforcement number of a graph has been studied in [9-12]. Recently, Muhiuddin et al. have studied various related concepts on graphs (see, e.g., $[13-17])$.

In $[18,19]$, a new domination parameter $\gamma_{\lambda}(G)$, where $0<\lambda<1$, was introduced and a study on $\gamma_{(1 / 2)}(G)$ had been initiated. In this paper, we introduce the concept of reinforcement number with respect to half-domination and initiate a study on this parameter.

\section{Definition and Examples}

If $G$ is a graph for every vertix $u$ of $G$, we define a map $f_{u}: V(G) \longrightarrow\{0,(1 / 2), 1\}$ as follows:

$$
f_{u}(v)= \begin{cases}1, & \text { if } \mathrm{d}(u, v) \leq 1, \\ \frac{1}{2}, & \text { if } \mathrm{d}(v, u)=2, \\ 0, & \text { otherwise, }\end{cases}
$$

for all $v \in V(G)$. The map $f_{u}$ is called the half-domination factor of $u$.

A subset $D$ of $V(G)$ is said to be a $(1 / 2)$-dominating set for $G$ if, for each $v \in V(G), \sum_{u \in D} f_{u}(v) \geq 1$. The minimum 
cardinality of a (1/2)-dominating set is called the $(1 / 2)$-domination number of $G$ and is denoted by $\gamma_{(1 / 2)}(G)$. A (1/2)-dominating set of $G$ with the minimum cardinality $\gamma_{(1 / 2)}(G)$ is said to be a $\gamma_{(1 / 2)}$-set of $G$. The parameter $\gamma_{(1 / 2)}(G)$ was introduced in [18] and bounds for $\gamma_{(1 / 2)}(G)$ are obtained in [18].

Theorem 1 (see [18]). If $G$ is a graph on $n$ vertices and $\Delta(G)=\Delta$, then

$$
\left\lceil\frac{n}{1+(1 / 2) \Delta+(1 / 2) \Delta^{2}}\right\rceil \leq \gamma_{(1 / 2)(G)} \leq n-\Delta .
$$

Theorem 2 (see [18]). If $G$ is a connected graph with $\Delta(G)$ $\leq n-3$, then $\gamma_{(1 / 2)}(G) \leq n-\Delta-1$. Moreover, trees $T$ with $\Delta(T) \leq n-3$, and $\gamma_{(1 / 2)}(G)=n-\Delta-1$ are characterized in [18].

Definition 1 (reinforcement number with respect to halfdomination). Let $G$ be a graph with $\gamma_{(1 / 2)}(G) \geq 2$. The reinforcement number with respect to half-domination is denoted by $\mathscr{R}_{(1 / 2)}(G)$ and is given by $\mathscr{R}_{(1 / 2)}(G)=\min \{A$ : $A \subseteq E \overline{(G)}$ and $\left.\gamma_{(1 / 2)}(G+A)<\gamma_{(1 / 2)}(G)\right\}$. For graphs $G$, with $\gamma_{(1 / 2)}(G)=1, \mathscr{R}_{(1 / 2)}(G)$ is not defined.

Remark 1. If $\gamma_{(1 / 2)}(G)=2$, then $\mathscr{R}_{(1 / 2)}(G)=n-\Delta-1$ (let $u$ be a vertex of $G$ with $\operatorname{deg}(u)=\Delta$. If $A=\left\{u v: v \notin N_{1}[u]\right\}$, then $\{u\}$ is a $\gamma_{(1 / 2)}$-dominating set for $\left.G+A\right)$.

$\mathscr{R}_{(1 / 2)}(G)$ for some standard graphs: here, we discuss path $P_{n}$ on $n$ vertices. As $\gamma_{(1 / 2)}\left(P_{n}\right)=2$, if $n=4,5,6,7$, it follows that $\mathscr{R}_{(1 / 2)}\left(P_{4}\right)=1, \mathscr{R}_{(1 / 2)}\left(P_{5}\right)=2, \mathscr{R}_{(1 / 2)}\left(P_{6}\right)=3$, and $\mathscr{R}_{(1 / 2)}\left(P_{7}\right)=4$.

\section{Theorem 3. If $n \geq 8$, then $\mathscr{R}_{(1 / 2)}\left(P_{n}\right)=$ $\{1$ if $n \equiv 0,1(\bmod 4)$ \\ 2 if $n \equiv 2,3(\bmod 4)$.}

Proof. $\gamma_{(1 / 2)}\left(P_{n}\right)=\lfloor n / 4\rfloor+1$ for all $n$. Let $P_{n}$ be $v_{1}, v_{2}, \ldots, v_{n}$. If $n=4 k$ or $4 k+1(k \geq 2)$, then $D=\left\{v_{2+4 i}: 0 \leq i \leq k-1\right\} \cup\left\{v_{4 k}\right\}$ is a $\gamma_{(1 / 2)}$-set of $P_{n}$. If $e=v_{2} v_{n}$, then $e \in E \overline{\left(P_{n}\right)}$ and $D-\left\{v_{4 k}\right\}$ is a half-dominating set for $P_{n}+e$. Thus, $\mathscr{R}_{(1 / 2)}\left(P_{n}\right)=1$ if $n=4 k$ or $4 k+1, k \geq 2$.

If $n=4 k+2$ or $4 k+3(k \geq 2)$, then $D=\left\{v_{2+4 i}: 0 \leq i \leq k\right\}$ is a $\gamma_{(1 / 2)}$-set of $P_{n}$. Let $e_{1}=v_{2} v_{7}$ and $e_{2}=v_{5} v_{4 k+2}$. Then, $D-$ $\left\{v_{6}\right\}$ is a half-dominating set for $P_{n}+A$, where $A=\left\{e_{1}, e_{2}\right\}$. Thus, $\mathscr{R}_{(1 / 2)}\left(P_{n}\right) \leq 2$. We claim that $\mathscr{R}_{(1 / 2)}\left(P_{n}\right) \geq 2$. If $\mathscr{R}_{(1 / 2)}\left(P_{n}\right)=1$, then there is a subset $S$ of $P(n)$ and an edge $e \in E\left(P_{n}\right)$ such that $|S|=k$ and $S$ is a half-dominating set for $P_{n}+e$. With respect the path $P_{n}$, either $N\left[u_{1}\right] \cap S \neq \phi$ or $N\left[u_{n}\right] \cap S \neq \phi$ (otherwise, $S$ cannot be a half-dominating set of $\left.P_{n}+e\right)$. In $P_{n}$ (by ignoring the contribution by the edge $e$ ), $f_{S}(V) \leq 4 k-(1 / 2)$. So, $n-f_{S}(V) \geq(5 / 2)$. But the contribution by the edge $e$ will be at most 2. Thus, $S$ cannot be a half-dominating set for $P_{n}+e$, which is a contradiction. Thus, $\mathscr{R}_{(1 / 2)}\left(P_{n}\right) \geq 2$, if $n=4 k+2$ or $4 k+3(k \geq 2)$.

One can easily prove the following theorem.

Theorem 4. If $C_{n}$ is a cycle on $n$-vertices $(n \geq 3)$, then

$$
\mathscr{R}_{(1 / 2)}\left(C_{n}\right)= \begin{cases}n-3, & \text { if } 3 \leq n \leq 8, \\ 1, & \text { if } n=9 \text { or } 4 k+1 \text { or } 4 k+2, k \geq 3, \\ 2, & \text { if } n=10 \text { or } 4 k \text { or } 4 k+3, k \geq 3 .\end{cases}
$$

Remark 2. We compare $\mathscr{R}_{(1 / 2)}(G)$, the reinforcement number of $G$ with respect to (1/2)-domination, and $\mathscr{R}_{t}(G)$, the reinforcement number with respect to the total-domination for some graphs, to show that there is no correlation between $\mathscr{R}_{(1 / 2)}(G)$ and $\mathscr{R}_{t}(G)$.

(a) If $G=P_{8}$, the path on eight vertices, $\gamma_{(1 / 2)}\left(P_{8}\right)=3$ and $\mathscr{R}_{(1 / 2)}\left(P_{8}\right)=1$, while $\gamma_{t}\left(P_{8}\right)=4$ and $\mathscr{R}_{t}\left(P_{8}\right)=3$

(b) If $G=P_{9}$, the path on nine vertices, $\gamma_{(1 / 2)}\left(P_{9}\right)=3$ and $\mathscr{R}_{(1 / 2)}\left(P_{9}\right)=1$, while $\gamma_{t}\left(P_{9}\right)=5$ and $\mathscr{R}_{t}\left(P_{9}\right)=1$

(c) If $G=C_{8}$, the cycle on eight vertices, $\gamma_{(1 / 2)}\left(C_{8}\right)=2$ and $\mathscr{R}_{(1 / 2)}\left(C_{8}\right)=5$, while $\gamma_{t}\left(C_{8}\right)=4$ and $\mathscr{R}_{t}\left(C_{8}\right)=2$

Theorem 5. If $T$ is a tree with $\gamma_{(1 / 2)}(T) \geq 3$, then $\mathscr{R}_{(1 / 2)}(T) \leq 3$.

Proof. As $\gamma_{(1 / 2)}(T) \geq 3, \operatorname{diam}(T) \geq 3$. Let $u_{1} u_{2}, \ldots, u_{l}$ be a diagonal path in $T$ (so $\left.\mathrm{d}\left(u_{1}, u_{l}\right)=\operatorname{diam}(T)\right)$. Let $D$ be a $\gamma_{(1 / 2)}$-set of $T$. We can assume that $u_{2} \in D$. If $u_{3} \in D$, let $e=u_{2} x$ for some $x \neq u_{2}, u_{3} \in D$. Then $D-u_{2}$ is a halfdominating set for $T+e$. Assume that $u_{3} \notin D$. If $\left|N\left(u_{3}\right) \cap D\right| \geq 3$, select $x, y \in D^{\prime}=D-u_{2}$. Let $A=$ $\left\{u_{2} x, u_{2} y\right\}$. Then $D^{\prime}=D-u_{2}$ is a half-dominating set of $T+A$. If $\left|N\left(u_{3}\right) \cap D\right| \leq 2$, find $x, y \in D^{\prime}=D-u_{2}$, such that $y \notin N\left(u_{3}\right)$. Let $e_{1}=u_{2} x, e_{2}=u_{2} y$, and $e_{3}=u_{3} y$. Then $D^{\prime}$ is a half-dominating set for $G+A$, where $A=\left\{e_{1}, e_{2}, e_{3}\right\}$. Thus, $\mathscr{R}_{(1 / 2)}(T) \leq 3$, for all trees $T$ with $\gamma_{(1 / 2)}(T) \geq 3$.

Remark 3. An example for a tree $T$, with $\mathscr{R}_{(1 / 2)}(T)=3$, is given in Figure 1.

\section{Bounds for $\mathscr{R}_{(1 / 2)}(G)$}

In this section, we obtain certain bounds for $\mathscr{R}_{(1 / 2)}(G)$. If $\gamma_{(1 / 2)}(G)=2$, then $\mathscr{R}_{(1 / 2)}(G)=n-\Delta(G)-1$. First, we have the following theorem.

Theorem 6. If $\gamma_{(1 / 2)}(G) \geq 2$, then $\mathscr{R}_{(1 / 2)}(G) \leq n-\Delta(G)-$ $\gamma_{(1 / 2)}(G)+1 \leq n-\Delta-1$.

Proof. Let $G$ be a graph with $\gamma_{(1 / 2)}(G)=k \geq 2$. Let $u$ be a vertex of degree $\Delta$. As $k \geq 2, V-N[u]$ contains at least $k-1$ vertices. Select a subset $S$ of $V(G)$ such that $|S|=k-1$ and $S \cap N[u]=\{u\}$. Let $A=\{u v: v \notin S \cup N[u]$ and $u v \in E \overline{(G)}\}$. Then $S$ is a dominating set of $G+A$. Thus, $\gamma_{(1 / 2)}(G+A) \leq$ $k-1$ and $\mathscr{R}_{(1 / 2)}(G) \leq|A| \leq n-\Delta-(k-1)=n-\Delta-k+$ $1 \leq n-\Delta-1$, as $k \geq 2$. 


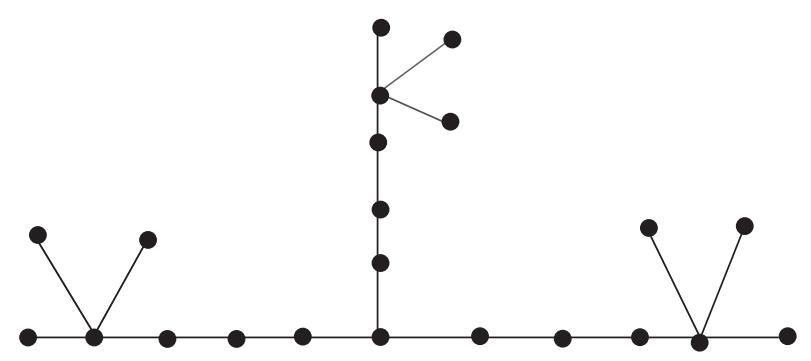

Figure 1: Example for a tree $T$, with $\gamma_{(1 / 2)}(t)=4$ and $\mathscr{R}_{(1 / 2)}(T)=3$.

Corollary 1. If $\gamma_{(1 / 2)}(G) \neq 1, \quad \mathscr{R}_{(1 / 2)}(G)=n-\Delta-1 \quad$ if $\gamma_{(1 / 2)}(G)=2$.

As a corollary, we get a better upper bound for $\gamma_{(1 / 2)}(G)$ which is given by inequality (1).

Corollary 2. For all graphs $G$ with $1 \leq \delta \leq \Delta \leq n-2$, $\gamma_{(1 / 2)}(G) \leq n-\Delta-\mathscr{R}_{(1 / 2)}(G)+1$.

Proof. Let $G$ be a connected graph with $\gamma_{(1 / 2)}(G)=k \geq 3$. Let $D=\left\{u_{1}, u_{2}, \ldots, u_{k}\right\}$ be a $\gamma_{(1 / 2)}$-set of $G$. Let $V_{0}=D$, $V_{1}=N_{1}(D)-D$, and $V_{2}=V-N_{1}[D]$. Note that if $v \in V_{2}$, then $v \in N_{2}\left(u_{i}\right) \cap N_{2}\left(u_{j}\right)$ for some $i, j, 1 \leq i<j \leq k$. Then, $V=V_{0} \cup V_{1} \cup V_{2}$ is a partition of $V(G)$. As $D$ is a $\gamma_{(1 / 2)}$-set of $G$, to each $u_{i}, D-u_{i}$ is not a half-dominating set of $G$ and hence the set $P\left(u_{i}, D\right)$, called private neighbors of $u_{i}$, consisting of all $v \in V(G)$ which are not half-dominated by the set $D-u_{i}$, is not empty. Note that $P\left(u_{i}, D\right)=$ $P_{1}\left(u_{i}, D\right) \cup P_{2}\left(u_{i}, D\right)$, where $P_{1}\left(u_{i}, D\right)=\{v \in V: N[v] \cap D=$ $\left\{u_{i}\right\}$ and $\left.\left|N_{2}(v) \cap D\right| \leq 1\right\}$ and $P_{2}\left(u_{i}, D\right)=\{v \in V: v \notin N$ $[D], u_{i} \in N_{2}(v)$ and $\left.\left|N_{2}(v) \cap D\right|=2\right\}$. As $P\left(u_{i}, D\right) \neq \phi$, either $P_{1}\left(u_{i}, D\right) \neq \phi$ or $P_{2}\left(u_{i}, D\right) \neq \phi$. We write $P\left(u_{i}\right), P_{1}\left(u_{i}\right)$, and $P_{2}\left(u_{i}\right)$ for $P\left(u_{i}, D\right), P_{1}\left(u_{i}, D\right)$, and $P_{2}\left(u_{i}, D\right)$, respectively. Now, we observe the following.

Observation 1. $|D|=\gamma_{(1 / 2)}(G) \leq \gamma(G) \leq\left|V_{1}\right|=\left|N_{1}(D)-D\right|$.

Proof. As $D$ is a $\gamma_{(1 / 2)}$-set of $G$, for each $u_{i}$, either $P_{1}\left(u_{i}\right) \neq \phi$ or $P_{2}\left(u_{i}\right) \neq \phi$, so $N\left(u_{i}\right)-D \neq \phi$ for each $i, 1 \leq i \leq k$. Hence, $V_{1}$ is a dominating set of $G$. Thus, $|D|=\gamma_{(1 / 2)}(G) \leq$ $\gamma(G) \leq\left|V_{1}\right|$.

Observation 2. If $\gamma_{(1 / 2)}(G) \geq 3$ and $|D|=\left|N_{1}(D)\right|$ for at least one $\gamma_{(1 / 2)}$-set $D$ of $G$, then $\mathscr{R}_{(1 / 2)}(G) \leq 2$.

Proof. If $|D|=\left|N_{1}(D)\right|$, then $N_{1}(D)=V_{1}$ is also a $\gamma_{(1 / 2)}$-set of $G$ and the maximum degree of the induced subgraph $\left\langle V_{1}\right\rangle \leq 1$ (if, for some $x \in V_{1}$, $\operatorname{deg}(x) \geq 2$ in $\left\langle V_{1}\right\rangle \leq 1$, then $V_{1}-x$ is also a half-dominating set of $G$, which is a contradiction as $\left.\left|V_{1}\right|=|D|=\gamma_{(1 / 2)}(G)\right)$. If $x y$ is an edge in $\left\langle V_{1}\right\rangle$, select a vertex $z \neq x, y \in V_{1}$ (note that $\left|V_{1}\right|=|D| \geq 3$ ). Then $V_{1}-\{x\}$ is a half-dominating set for $G+e$, where $e=x y$. Thus, in this case, $\mathscr{R}_{(1 / 2)}(G)=1$. If $V_{1}$ is an independent set in $G$, select $x, y, z \in V_{1}$. Let $A=\{x y, x z\} \subseteq E(\bar{G})$. Then, $V_{1}-\{x\}$ is a half-dominating set for $G+A$. Thus, $\mathscr{R}_{(1 / 2)}(G) \leq|A|=2$.
Observation 3. If $V_{2}=\phi$ for some $\gamma_{(1 / 2)}$-set $D$ of $G$, then $\mathscr{R}_{(1 / 2)}(G) \leq 2$.

Proof. If $V_{2}=\phi, P_{2}\left(u_{1}\right)=\phi$ and hence $D-\{u\}$ is a halfdominating set of $G+\left\{u_{1} u_{2}, u_{1} u_{3}\right\}$.

Observation 4. If $G$ is a connected graph with $\gamma_{(1 / 2)}(G)=k \geq 3$, then $\mathscr{R}_{(1 / 2)}(G) \leq(2 n / k)-2 \leq(2 n / 3)-2$.

Proof. If $P_{2}\left(u_{i}\right)=\phi$ for any $i, 1 \leq i \leq k$ (with respect to some $\gamma_{(1 / 2)}$-set $D$ of $\left.G\right)$, then, by our Observation $1, \mathscr{R}_{(1 / 2)}(G) \leq 2$ and so $\mathscr{R}_{(1 / 2)}(G) \leq(2 n / k)-2$ (as $G$ is connected, $\left.2 k \leq n\right)$. If $|D|=\left|V_{0}\right|=\left|V_{1}\right|$, then also $\mathscr{R}_{(1 / 2)}(G) \leq 2$ and hence the result is true. So assume that $|D|<\left|V_{1}\right|$ and $P_{2}\left(u_{i}\right) \neq \phi$ for all $i, 1 \leq i \leq k$. If $x \in P_{2}\left(u_{i}, D\right)$, for some $i$, then $x \in P_{2}\left(u_{j}, D\right)$ for exactly one $j \neq i$. Thus, each $x \in P_{2}\left(u_{j}, D\right)$ is counted twice in $\sum_{i=1}^{k} P_{2}\left(u_{i}, D\right)$. As $\left|V_{1}\right| \geq k+1,\left|V_{2}\right|=V-N_{1}[D] \leq$ $n-(2 k+1)$. So, $\sum\left|P_{2}\left(u_{i}\right)\right| \leq 2\left|V_{2}\right| \leq 2 n-4 k-2$ and hence we can find one $u_{j}$ such that $\left|P_{2}\left(u_{j}\right)\right| \leq((2 n-4 k-2) / k)$. To each vertex $w \in P_{2}\left(u_{j}\right)$, select a vertex $x \in N_{1}\left(u_{j}\right)$ such that $x w$ is an edge in $G$, and select a vertex $u_{i}$ such that $w \notin N_{2}\left(u_{i}\right)$ (since $\left|N_{2}(w) \cap D\right|=2$, there is a vertex $u_{i} \in D$ such that $\left.w \notin N_{2}\left(u_{i}\right)\right)$, and let $e_{w}=x v \in E \overline{(G)}$. Let $A=\left\{e_{w}: w \in P_{2}\left(u_{j}\right)\right\} \cup\left\{u_{j} u_{i}, u_{j} u_{i_{2}}\right\}$, where $i_{1} \neq i_{2}$ and $j \notin$ $\left\{i_{1}, i_{2}\right\}$. Then, $\gamma_{(1 / 2)}(G+A) \leq k-1$ and hence $\mathscr{R}_{(1 / 2)}(G) \leq$ $|A|+2=\left|P_{2}\left(u_{j}\right)\right|+2 \leq((2 n-4 k-2) / k)+2=((2(n-1)) /$ k) -2 .

Observation 5. If $\left|N_{1}\left(u_{i}\right)\right|=1$, for some $u_{i}$ in a $\gamma_{(1 / 2)}$-set $D$, then $\mathscr{R}_{(1 / 2)} \leq 2$.

Observation 6. For a connected graph $G$, with radius of $G \geq 3$ and $\gamma_{(1 / 2)}(G) \geq 3, \mathscr{R}_{(1 / 2)}(G) \leq n-\Delta-\delta-\gamma_{(1 / 2)}+1$ (radius of $G \geq 3 \Longrightarrow$ to each vertex $x \in v$, there is a vertex $y$ such that $\mathrm{d}(x, y) \geq 3)$.

Proof. Let $k=\gamma_{(1 / 2)}(G)$. Let $v_{1}$ be a vertex of $G$ with $\operatorname{deg}\left(v_{1}\right)=\Delta$. Let $v_{2}$ be a vertex such that $\mathrm{d}\left(v_{1}, v_{2}\right) \geq 3$. Then, $N_{1}\left[v_{1}\right] \cap N_{1}\left[v_{2}\right]=\phi$. Let $B=V-\left(N_{1}\left[v_{1}\right] \cup N_{1}\left[v_{2}\right]\right)$. Then, $B$ contains at least $k-3$ vertices. Select $k-2$ vertices $v_{3}, v_{4}, \ldots, v_{k-1}$ in $B$. Let $A=\left\{v_{1} x: x \in B-\left\{v_{3}, \ldots, v_{k-1}\right\}\right.$. Note that $A \subset E \overline{(G)}$. Now, $S=\left\{v_{i}: 1 \leq i \leq k-1\right\}$ is a halfdominating set for $G+A$. So $\mathscr{R}_{(1 / 2)}(G) \leq n-\Delta-\delta-k+1$, where $k=\gamma_{(1 / 2)}(G)$.

Remark 4. The upper bound given in Observation 6 is attained by the graph in Figure 2. For this graph, $n=9$, $\gamma_{(1 / 2)}(G)=3, \Delta=4, \delta=2$, and $\mathscr{R}_{(1 / 2)}(G)=1$.

Remark 5. For a connected graph $G$ with radius $\geq 3$ and $\gamma_{(1 / 2)}(G) \geq 3$, Observation 6 yields a better bound for $\gamma_{(1 / 2)}(G)$ than the bound given by inequality (1). $\gamma_{(1 / 2)}(G) \leq n-\Delta-\delta-\mathscr{R}_{(1 / 2)}(G)+1$. In the following theorem, we obtain an upper bound for $\mathscr{R}_{(1 / 2)}(G)$ in terms of $\Delta(G)$. 


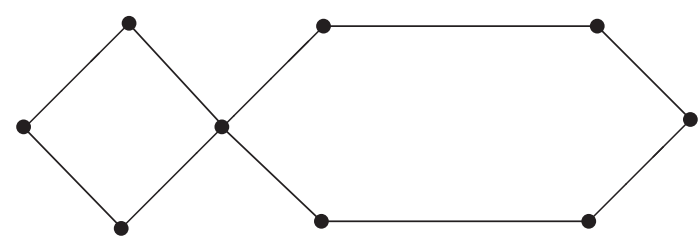

Figure 2: A group $G$ for which $\mathscr{R}_{(1 / 2)}(G)=n-\Delta-\delta-$ $\gamma_{(1 / 2)}(G)+1$.

Theorem 7. For any graph $G$, with $\gamma_{(1 / 2)}(G) \geq 3$, $\mathscr{R}_{(1 / 2)}(G) \leq 2 \Delta$.

Proof. Let $D$ be a $\gamma_{(1 / 2)}$-set of $G$. Select three vertices $u, v, w$ in $D$. Let $A=\{x v, x w: x \in N(u)\} \cap \overline{E(G)}$. Then, $|A| \leq 2 \operatorname{deg}(u) \leq 2 \Delta(G)$ and $D-\{u\}$ is a half-dominating set of $G+A$; thus, $\mathscr{R}_{(1 / 2)}(G) \leq 2 \Delta$.

Remark 6. There are graphs $G$, for which $\gamma_{(1 / 2)}(G) \geq 3$ and $\mathscr{R}_{(1 / 2)}(G)=2 \Delta$.

Example 1. (Example for a graph $G$ with $\gamma_{(1 / 2)}(G) \geq 3$ and $\left.\mathscr{R}_{(1 / 2)}(G)=2 \Delta\right)$. Consider the graph $G$ given in Figure 3 . First, we note that for this graph $n=21, \Delta=3, \delta=2$, and $\left(n /\left(1+(\Delta / 2)+\left(\Delta^{2} / 2\right)\right)\right)=\gamma_{(1 / 2)}(G)=3 .\left(\gamma_{(1 / 2)}(G)\right.$ attains its lower bound given in (1). The set $D=\{a, b, c\}$ is the unique $\gamma_{(1 / 2)}$-set of $G$ (for any vertex $s \in V(G), f_{s}(V) \leq(n /(1+(\Delta /$ $\left.\left.2)+\left(\Delta^{2} / 2\right)\right)\right)=7$ and $f_{s}(V)=7$ if $s \in D$ and $f_{s}(V)<7$ for all $s \notin D$. If $D^{\prime}$ is any three-element subset of $V$, other than $D$, then $\left.f_{D^{\prime}}(V)<21\right) . V(G)=V_{1} \cup V_{2} \cup V_{3}$, where $V_{1}=\{a, b$, c\}, $V_{2}=\left\{x_{i}, y_{i}, z_{i}: 1 \leq i \leq 3\right\}$, and $V_{3}=\left\{u_{i j}, v_{i j}, w_{i j}: 1 \leq\right.$ $i<j \leq 3\}$. Assume that $B$ is a two-element subset of $V$ and $A \subset E \overline{(G)}$ such that $B$ is a half-domination set of $G+A$. In order to show that $\mathscr{R}_{(1 / 2)}(G)=6$, it is enough to prove that $|A| \geq 6$. Claim: $|A| \geq 6$.

In our further discussion, we consider the following notations.

If $e \in A$, we write $T_{e}(s)$, where $s \in V(G)$, and the number $T_{e}(s)=\left(\sum_{t \in B} f_{t}(s)\right.$ in $\left.G+e\right)-\left(\sum_{t \in B} f_{t}(s)\right.$ in $\left.G\right)$. We say that the edge $e \in A$ contributes the value $\lambda$ to a vertex $s$ of $G$ if $\lambda=T_{e}(s)$. Similarly, we say that $e$ contributes the value $\lambda$ to $V(G)$ if $\lambda=T_{e}(V)=\sum_{s \in V} T_{e}(s)$. If $A^{\prime} \subset A$, we define $\quad T_{A^{\prime}}(s)=\left(\sum_{t \in B} f_{t}(s)\right.$ in $\left.G+A\right)-\left(\sum_{t \in B} f_{t}(s)\right.$ in $\left.G\right)$ and $T_{A^{\prime}}(V)=\sum_{s \in V} T_{A^{\prime}}(s)$. We note that $T_{e}(s) \leq 1$ for all $e \in A$ and $s \in V(G)$. We can also assume that every edge $e$ in $A$ has one end in $B$. Now, we establish our claim $|A| \geq 6$ by considering various possibilities for $B$ (for each possibility, we consider one subcase; other subcases can be proved similarly):

Case (i): $B \subset V_{1}$. Assume that $B=\{a, b\}$. Then $f_{B}(s)=$ $(1 / 2)$ if $s \in\left\{u_{13}, v_{13}, w_{13}, u_{23}, v_{23}, w_{23}\right\}$. If, for an edge $e \in A, T_{e}\left(s_{0}\right) \geq(1 / 2)$ for one of the elements $s_{0}$ in $\left\{u_{13}, v_{13}, w_{13}\right\}$, then $T_{s}(s)=0$ for $s \neq s_{0} \in\left\{u_{13}, v_{13}, w_{13}\right\}$ and $b$ is one of the ends of $e$. Similarly, if $T_{e}\left(s_{0}\right) \geq(1 / 2)$

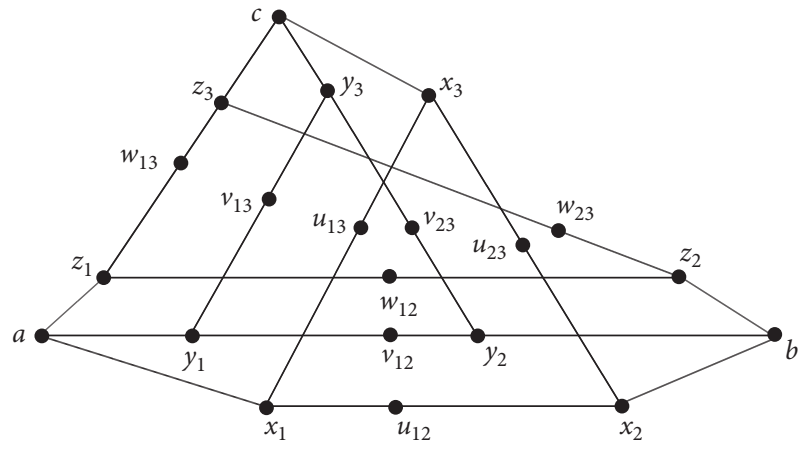

Figure 3: A graph $G$ with $\gamma_{(1 / 2)}(G)=3$ and $\mathscr{R}_{(1 / 2)}(G)=2 \Delta=6$.

for one $s_{0} \in\left\{u_{23}, v_{23}, w_{23}\right\}$, then $T_{e}(s)=0$ for $s \neq s_{0} \in\left\{u_{23}, v_{23}, w_{23}\right\}$ and $a$ is one of the ends of $e$. Thus, $|A| \geq 6$.

Case (ii): $B \subset V_{2}$. Assume that $B=\left\{x_{1}, z_{2}\right\}$. Then $f_{B}(s)=(1 / 2)$ if $s \in\left\{y_{1}, y_{2}, x_{3}, z_{3}\right\}$ and $f_{B}(s)=0$ if $s \in\left\{c, y_{3}, u_{23}, w_{13}, v_{12}, v_{13}, v_{23}\right\}$. Let $A^{\prime} \subset A$ be such that $T_{A^{\prime}}\left(y_{1}\right), T_{A^{\prime}}\left(y_{2}\right) \geq(1 / 2) ; T_{A^{\prime}}(s) \geq 1$ for $s \in\left\{v_{12}, v_{13}\right.$, $\left.v_{23}, z_{3}\right\}$; and $T_{e}(Y) \geq(1 / 2)$ for every $e \in A^{\prime}$, where $Y=\left\{y_{1}, y_{2}, y_{3}, v_{12}, v_{13}, v_{23}\right\}$. Then $\left|A^{\prime}\right| \geq 3 ; T_{A^{\prime}}(c) \geq$ (1/2) only if $\left|A^{\prime}\right| \geq 4$. If $\left|A^{\prime}\right|=4, A-A^{\prime}$ contains at least two edges $e_{1}$ and $e_{2}$ so that $T_{e_{1}}\left(u_{23}\right) \geq(1 / 2)$ and $T_{e_{2}}\left(w_{13}\right) \geq(1 / 2)$. So, in this case, $|A| \geq 6$. If $\left|A^{\prime}\right|=3, A-$ $A^{\prime}$ contains a set $A^{\prime \prime}$ of at least three edges such that $T_{A^{\prime \prime}}(s) \geq 1$, where $s \in\left\{c, u_{23}, v_{23}\right\}$. Thus, in this case, also $|A| \geq 6$.

Case (iii): $B \subset V_{3}$. Assume that $B=\left\{u_{12}, w_{13}\right\}$. Then $f_{B}(s)=(1 / 2) \quad$ if $\quad s \in\left\{u_{13}, u_{23}, w_{12}, w_{23}, b, c\right\} \quad$ and $f_{B}(s)=0$ if $s \in\left\{y_{1}, y_{2}, y_{3}, v_{12}, v_{13}, v_{23}, x_{3}, z_{2}\right\}$. If $A^{\prime} \subset A$ such that $T_{A^{\prime}}(s) \geq 1$ for $s \in\left\{y_{1}, y_{2}, y_{3}, v_{12}\right.$, $\left.v_{13}, v_{23}\right\}$, then $\left|A^{\prime}\right| \geq 4\left(A^{\prime}\right.$ contains at least two edges with one end at $u_{12}$ and at least two edges with one end at $\left.w_{13}\right) . A-A^{\prime}$ contains an edge $e_{1}$ with one end at $w_{13}$ and $T_{e_{1}}\left(u_{13}\right) \geq(1 / 2)$ and contains another edge $e_{2}$ with one end at $u_{12}$ and $T_{e}\left(w_{23}\right) \geq(1 / 2)$. Thus, $|A| \geq 6$.

Case (iv): $B \cap V_{1} \neq \phi$ and $B \cap V_{2} \neq \phi$. Assume that $B=\left\{a, x_{2}\right\}$. Then, $f_{B}(s)=(1 / 2)$ if $s \in\left\{u_{13}, v_{13}, w_{13}\right.$, $\left.v_{12}, w_{12}, x_{2}, y_{2}, z_{2}\right\}$ and $f_{B}(s)=0$ if $s \in\left\{c, y_{3}, z_{3}, w_{23}\right.$, $\left.v_{23}\right\}$. The set $A$ contains a subset $A^{\prime}$, with $\left|A^{\prime}\right|=3$, $T_{A^{\prime}}(s) \geq(1 / 2)$ for $s \in\left\{u_{12}, v_{13}, w_{13}\right\}$, and each edge in $A^{\prime}$ has one end at $x_{2}$. Note that $T_{A^{\prime}}(c) \leq(1 / 2)$, either $T_{A^{\prime}}\left(w_{23}\right)=0$ or $T_{A^{\prime}}\left(w_{12}\right)=0$, and either $T_{A^{\prime}}\left(v_{12}\right)=0$ or $T_{A^{\prime}}\left(v_{23}\right)=0$. So, there is a set $A^{\prime \prime}$ contained in $A-$ $A^{\prime}$ with $\left|A^{\prime \prime}\right| \geq 3$ such that $T_{B \cup A^{\prime} \cup A^{\prime \prime}}(s) \geq 1$ for $s \in\left\{c, w_{23}, v_{12}, w_{12}, w_{23}\right\}$. Thus, $6 \leq\left|A^{\prime}\right|+\left|A^{\prime \prime}\right| \leq|A|$.

Case (v): $B \cap V_{1} \neq \phi$ and $B \cap V_{3} \neq \phi$. Assume that $B=\left\{a, w_{23}\right\}$. Then, $f_{B}(s)=(1 / 2)$ if $s \in\left\{u_{12}, u_{13}, v_{12}\right.$, $\left.v_{13}, b, c\right\}$ and $f_{B}(s)=0$ if $s \in\left\{x_{2}, x_{3}, y_{2}, y_{3}, u_{23}, v_{23}\right\}$. There are edges $e_{1}$ and $e_{2}$ in $A$ with one end at $a$ and 
$T_{e_{1}}(c) \geq(1 / 2)$. Note that $T_{e_{1}}\left(u_{23}\right)+T_{e_{2}}\left(u_{23}\right) \leq(1 / 2)$ and $T_{e_{1}}\left(v_{23}\right)+T_{e_{2}}\left(v_{23}\right) \leq(1 / 2)$. There are edges $e_{3}$ and $e_{4}$ in $A$, with one end at $w_{23}$ and $T_{e_{3}}\left(u_{13}\right) \geq(1 / 2)$; $T_{e_{4}}\left(v_{13}\right) \geq(1 / 2)$. Now either $T_{e_{3}}\left(u_{23}\right)=0$ or $T_{e_{3}}\left(u_{12}\right)=0$, and either $T_{e_{4}}\left(v_{23}\right)=0$ or $T_{e_{4}}\left(v_{12}\right)=0$. As $A_{1}=\left\{e_{1}, e_{2}, e_{3}, e_{4}\right\} \subset A \quad$ and $T_{A}(s) \geq(1 / 2)$ for $s \in\left\{c, b, u_{13}, u_{12}, v_{12}, v_{13}\right\} \quad$ and $T_{A}(s) \geq 1 \quad$ for $s \in\left\{u_{23}, v_{23}\right\}$, we have $|A| \geq 6$.

Case (vi): $B \cap V_{2} \neq \phi$ and $B \cap V_{3} \neq \phi$. Let $B=\left\{x_{1}, v_{23}\right\}$. Then $f_{B}(s)=(1 / 2)$ for $s \in\left\{c, b, u_{13}, u_{12}, v_{12}, v_{13}\right\}$ and $f_{B}(s)=0$ if $s \in\left\{z_{2}, z_{3}, w_{12}, w_{23}, w_{13}, u_{23}\right\}$. The set $A$ should contain at least three edges to attribute positive values to $z_{1}, z_{2}, z_{3}, w_{12}, w_{23}$; it should contain at least two edges with one end at $v_{23}$ to contribute positive values to $y_{1}$ and $x_{3}$ and one edge with one end at $x_{1}$ to contribute a positive value to $v_{13}$. Thus, $|A| \geq 6$. The upper bound $2 \Delta$ for $\mathscr{R}_{(1 / 2)}(G)$ can be reduced further if $\Delta<\gamma_{(1 / 2)}(G)$.

Theorem 8. If $\gamma_{(1 / 2)}(G) \geq 3$ and $\Delta(G)<\gamma_{(1 / 2)}(G)$, then

$$
\mathscr{R}_{(1 / 2)}(G) \leq \begin{cases}\Delta, & \text { if } \Delta(G)<\gamma_{(1 / 2)}(G)-1, \\ \Delta+1, & \text { if } \Delta(G)=\gamma_{(1 / 2)}(G)-1 .\end{cases}
$$

Proof. Let $\gamma_{(1 / 2)}(G)=k \geq 3$. Let $D=\left\{u_{1}, u_{2}, \ldots, u_{k}\right\}$ be a $\gamma_{(1 / 2)}$-set of $G$. Let $N_{1}\left(u_{1}\right)-D=\left\{v_{1}, v_{2}, \ldots, v_{l}\right\}$. Let $1 \leq j \leq l$. If $P_{2}\left(u_{1}\right) \cap N_{1}\left(v_{j}\right)=\phi$, select one $i_{j}\left(2 \leq i_{j} \leq k\right)$ such that $u_{i_{j}} v_{j} \in E \overline{(G)}$. If $\quad P_{2}\left(u_{1}\right) \cap N_{1}\left(v_{j}\right) \neq \phi$, then either $N_{1}\left(v_{j}\right) \cap D=\left\{u_{1}\right\} \quad$ or $\quad\left|N_{1}\left(v_{j}\right) \cap D\right|=2$. If $\quad N_{1}\left(v_{j}\right) \cap$ $D=\left\{u_{1}\right\}$, then, for each vertex $w \in P_{2}\left(u_{1}\right) \cap N_{1}\left(v_{j}\right)$, $\mid\left\{i: i \neq 1\right.$ and $\left.w \in N_{2}\left(u_{i}\right)\right\} \mid=1$. So, we can select one $i_{j}\left(2 \leq i_{j} \leq k\right)$ such that $P_{2}\left(u_{1}\right) \cap N_{1}\left(v_{j}\right) \cap N_{2}\left(u_{i_{j}}\right)=\phi$ (this is possible since $\left.\left|P_{2}\left(u_{1}\right) \cap N_{1}\left(v_{j}\right)\right| \leq \delta-1<\gamma_{(1 / 2)}(G)-1\right)$. Thus, to each $v_{j},(1 \leq j \leq l)$, we have selected one $u_{i_{j}} \in D-\left\{u_{1}\right\}$. If $\Delta(G)<\gamma_{(1 / 2)}(G)-1$, we can select $u_{i_{l}}$ such that $u_{i_{l}} \neq u_{i_{1}}$. Let $A=\left\{v_{j} u_{i_{j}}: 1 \leq j \leq l\right\}$. If $\Delta(G)<\gamma_{(1 / 2)}(G)-1$, $D-u_{1}$ is a half-dominating set of $G+A$. If $\Delta(G)<\gamma_{(1 / 2)}(G)-1$, then $D-u_{1}$ is a half-dominating set of $G+\left(A+e_{1}\right)$, where $e_{1}=u_{1} u_{j}$ for some $u_{j} \notin N\left(u_{1}\right)$ (as $D-u_{1}$ is not a half-dominating set of $\left.G, N\left(u_{1}\right) \neq D-u_{1}\right)$. Thus, $\mathscr{R}_{(1 / 2)}(G) \leq l \leq \Delta$ if $\Delta(G)<k-1$ and $\mathscr{R}_{(1 / 2)}(G) \leq l+1 \leq \Delta+1$ if $\Delta(G)=k-1$. In the following theorem, we obtain a lower bound for $\mathscr{R}_{(1 / 2)}(G)$ of some classes of graphs.

Theorem 9. If $G$ is a connected graph with $\gamma_{(1 / 2)}(G)=k \geq 3$ and the order $n=k\left(1+(\Delta / 2)+\left(\Delta^{2} / 2\right)\right)$, then $\mathscr{R}_{(1 / 2)}(G) \geq \Delta$.

Proof. If $S=\left\{u_{1}, u_{2}, \ldots, u_{k-1}\right\}$ is a set of vertices of $G$, then $f_{s}(V) \leq(k-1)\left(1+(\Delta / 2)+\left(\Delta^{2} / 2\right)\right)$. If $S$ is a halfdominating set of $G+A$, where $A \subseteq E \overline{(G)}$, then $A$ should contribute at least $1+(\Delta / 2)+\left(\Delta^{2} / 2\right)$ to the total $\sum f_{s}(V)$ in $G+A$. But $T_{e}$ is an edge $e$ in $A$ which can contribute at most
$1+(\Delta / 2)$. So, $|A| \geq\left(\left(1+(\Delta / 2)+\left(\Delta^{2} / 2\right)\right) / 1+(\Delta / 2)\right)=\Delta-$ $1+(2 /(1+\Delta))$. Hence, $|A| \geq \Delta$ whenever $\gamma_{(1 / 2)}(G+A) \leq$ $k-1$. Thus, $\mathscr{R}_{(1 / 2)}(G) \geq \Delta$.

Corollary 3. If, for a graph $G$ with $\gamma_{(1 / 2)}(G)=k \geq 3, \Delta<k-$ 1 and $n=k\left(1+(\Delta / 2)+\left(\Delta^{2} / 2\right)\right)$, then $\mathscr{R}_{(1 / 2)}(G)=\Delta$.

Example 2. If $\Delta=2, \gamma_{(1 / 2)}(G)=k \geq 4$, and $n=k(1+(\Delta / 2)+$ $\left.\left(\Delta^{2} / 2\right)\right)$ for a connected graph $G$, then $G=C_{4 k}$ for $k \geq 4$. We know that $\mathscr{R}_{(1 / 2)}\left(C_{4 k}\right)=2$ for all $k \geq 3$.

Remark 7. For all $k \geq 3$ and $\Delta<k-1$, we can construct graphs $G$ with $\gamma_{(1 / 2)}(G)=k, \quad \Delta(G)=\Delta, \quad$ and $n=k\left(1+(\Delta / 2)+\left(\Delta^{2} / 2\right)\right)$. Now, we list some properties of graphs $G$ with $\gamma_{(1 / 2)}(G) \geq 3$ and $\mathscr{R}_{(1 / 2)}(G)=2 \Delta$. Let $D=$ $\left\{u_{1}, u_{2}, \ldots, u_{k}\right\}$ be any $\gamma_{(1 / 2)}$-set of $G\left(\right.$ where $\left.k=\gamma_{(1 / 2)}(G)\right)$. Then,

(1) $k=\gamma_{(1 / 2)} \leq \Delta$

(2) $\operatorname{deg}\left(u_{i}\right)=\Delta$ for all $i, 1 \leq i \leq k$

(3) $N\left[u_{i}\right] \cap N\left[u_{j}\right]=\phi$ for all $1 \leq i<j \leq k$

(4) $\left|P_{2}\left(u_{i}\right) \cap N_{1}(v) \cap N_{2}\left(u_{j}\right)\right| \neq 0$ for all $i \neq j \in$ $\{1,2, \ldots, k\}$ and for all $v \in N_{1}\left(u_{i}\right)$. So $\left|P_{2}\left(u_{i}\right) \cap N_{1}(v)\right| \geq k-1 \forall 1 \leq i \leq k$ and $v \in N_{1}\left(u_{i}\right)$

Problem 1. Characterize the graphs $G$ with $\gamma_{(1 / 2)}(G) \geq 3$ and $\mathscr{R}_{(1 / 2)}(G)=2 \Delta(G)$.

\section{Nordhaus-Gaddum-Type Results}

If $G$ is a graph with $1 \leq \delta \leq \Delta \leq n-2$, both $\mathscr{R}_{(1 / 2)}(G)$ and $\mathscr{R}_{(1 / 2)} \overline{(G)}$ are defined. In this section, we obtain certain bounds for the sum $\mathscr{R}_{(1 / 2)}(G)+\mathscr{R}_{(1 / 2)} \overline{(G)}$. We use the following notations:

If $\quad v \in V(G)$, then $\overline{N_{1}}(v)=\{u \in(V / v) \neq u$ and $v u \in E \overline{(G)}\}$ and $\overline{N_{2}}(v)=\{u \in(V / \mathrm{d}(u, v))=2$ in $\bar{G}\}$. Also, $\bar{\delta}$ and $\bar{\Delta}$ denote the minimum degree and maximum degree of $\bar{G}$, respectively. Note that $\bar{\delta}=n-\Delta(G)-1$ and $\bar{\Delta}=n-\delta(G)-1$.

Theorem 10. If $1 \leq \delta(G) \leq \Delta(G) \leq n-2$, then $\mathscr{R}_{(1 / 2)}(G)+\mathscr{R}_{(1 / 2)}(G) \leq n-(\Delta-\delta)-1 \leq n-1$.

Proof. By Theorem 6, $\mathscr{R}_{(1 / 2)}(G) \leq n-\Delta(G)-1$ and $\mathscr{R}_{(1 / 2)} \overline{(G)} \leq n-\bar{\Delta}-1$.

$$
\begin{aligned}
\mathscr{R}_{(1 / 2)}(G)+\mathscr{R}_{(1 / 2)} \overline{(G)} & \leq n-\Delta(G)-1+\delta(G) \\
& =n-(\Delta-\delta)-1 \leq n-1 .
\end{aligned}
$$

Corollary 4. If $1 \leq \delta(G) \leq \Delta(G) \leq n-2, \quad \mathscr{R}_{(1 / 2)}(G)+$ $\mathscr{R}_{(1 / 2)} \overline{(G)}=n-1$ if $G$ is regular and $\gamma_{(1 / 2)}(G)=\gamma_{(1 / 2)} \overline{(G)}=2$. Also, $\mathscr{R}_{(1 / 2)}(G)+\mathscr{R}_{(1 / 2)} \overline{(G)}=n-2$ if $\Delta(G)=\delta(G)+1$ and $\gamma_{(1 / 2)}(G)=\gamma_{(1 / 2)} \overline{(G)}=2$.

Theorem 11. If $1 \leq \delta(G) \leq \Delta(G) \leq n-2$, then $\mathscr{R}_{(1 / 2)}(G)+$ $\mathscr{R}_{(1 / 2)} \overline{(G)} \leq n-(\Delta-\delta)-\gamma_{(1 / 2)}(G)+1$. 
Proof. By Theorem 6, $\mathscr{R}_{(1 / 2)}(G) \leq n-\Delta(G)-\gamma_{(1 / 2)}(G)+1$ and $\quad \mathscr{R}_{(1 / 2)} \overline{(G)} \leq n-\bar{\Delta}-1=\delta(G)$. So, $\quad \mathscr{R}_{(1 / 2)}(G)+$ $\mathscr{R}_{(1 / 2)} \overline{(G)} \leq n-(\Delta-\delta)-\gamma_{(1 / 2)}(G)+1$. Now, we show that if $\gamma_{(1 / 2)}(G) \geq 3$, then either $\gamma_{(1 / 2)}(G)=2$ or $\gamma_{(1 / 2)} \overline{(G)}=2$.

Theorem 12. If $1 \leq \delta \leq \Delta \leq n-2$, then either $\gamma_{(1 / 2)}(G)=2$ or $\gamma_{(1 / 2)} \overline{(G)}=2$.

Proof. Assume that $\gamma_{(1 / 2)}(G) \geq 3$. Let $D=\left\{u_{1}, \ldots, u_{k}\right\}$ be a $\gamma_{(1 / 2)}$-set of $G$. Let $V=V_{0} \cup V_{1} \cup V_{2}$, where $V_{0}=D$, $V_{2}=V-N[D]$, and $V_{1}=V-\left(V_{0} \cup V_{2}\right)$. We claim that $\gamma_{(1 / 2)} \overline{(G)}=2\left(\right.$ as $\left.1 \leq \delta(G), \gamma_{(1 / 2)} \overline{(G)} \neq 1\right)$.

Case (i): $\left|\cup_{i=1}^{k} P_{2}\left(u_{i}, D\right)\right| \geq 2$. Select $x \neq y \in \cup P_{2}\left(u_{i}, D\right)$. Assume that $x \in P_{2}\left(u_{1}, D\right) \cap P_{2}\left(u_{2}, D\right)$ and $y \in P_{2}\left(u_{i_{1}}, D\right) \cap P_{2}\left(u_{i_{2}}, D\right)$, where $i_{1} \neq i_{2}\left(\left\{i_{1}, i_{2}\right\}\right.$ may be the set $\{1,2\})$. Clearly, $D=V_{0} \subset \overline{N_{1}}(x) \cap \overline{N_{1}}(y)$, and $V_{2} \subset \overline{N_{1}}(D)$. So, $V_{2}-\{x, y\} \subset \overline{N_{2}}(x) \cap \overline{N_{2}}(y)$. Let $v \in V_{1}$. Then $v \in N_{1}\left(u_{j}\right)$ for some $j$. If $j \notin\{1,2\}$, then either $v \in \overline{N_{1}}(x)$ or $v \in \overline{N_{1}}\left(u_{1}\right)$ and hence either $v \in \overline{N_{1}}(x)$ or $v \in \overline{N_{2}}(x) \cap \overline{N_{2}}(y)$. If $j \in\{1,2\}$, then either $v \in \overline{N_{1}}(x)$ or $v \in \overline{N_{1}}\left(u_{3}\right)$. Thus, if $\left|\cup_{i=1}^{k} P_{2}\left(u_{i}, D\right)\right| \geq 2,\{x, y\}$ is a half-dominating set for $\bar{G}$.

Case (ii): $\left|\cup_{i=1}^{k} P_{2}\left(u_{i}, D\right)\right|=1$. Let $\cup P_{2}\left(u_{i}, D\right)=\{x\}$. Assume that $x \in N_{2}\left(u_{1}\right) \cap N_{2}\left(u_{2}\right)$. Then, $x \notin N_{2}\left(u_{i}\right)$ for all $i \geq 3$. As $P_{2}\left(u_{3}, D\right)=\phi, P_{1}\left(u_{3}, D\right) \neq \phi$. Select $y \in P_{1}\left(u_{3}, D\right)$. Then, $V_{0} \subset \overline{N_{1}(x)}$, and $V_{0}-u_{3} \subset$ $\overline{N_{1}}(y) . \quad V_{2} \subset \overline{N_{2}}(x) \cap \overline{N_{2}}(y)$ as $V_{2} \subset \overline{N_{2}}\left(u_{3}\right)$. Let $v \neq y \in V_{1}$ and $v \in N_{1}\left(u_{j}\right)$ for some $j$. If $j \notin\{1,2\}$, $v \in \overline{N_{1}(x)}$. If $j \in\{1,2\}$, say $j=1$; then either $v \in \overline{N_{1}(y)}$ or $v \in \overline{N_{1}\left(u_{2}\right)}$. Thus, in this case, $\{x, y\}$ is a halfdominating set of $\bar{G}$.

Case (iii): $P_{2}\left(u_{i}, D\right)=\phi$ for all $i$. Then, $P_{1}\left(u_{i}, D\right) \neq \phi$ for all $i$. Let $x \in P_{1}\left(u_{1}, D\right)$, and $y \in P_{1}\left(u_{2}, D\right)$. Then, $u_{1} \in \overline{N_{1}}(y), \quad u_{2} \in \overline{N_{1}}(x), \quad$ and $\quad u_{i} \in \overline{N_{1}}(x) \cap$ $\overline{N_{1}}(y) \forall i \geq 3 . \quad V_{2} \subset \overline{N_{1}}\left(u_{3}\right) \subset \cap \overline{N_{2}(x)} \cap \overline{N_{2}}(y)$. Let $v \neq x, y \in V_{1}$. Then, either $v \in \overline{N_{1}}(x)$ or $v \in \overline{N_{1}}\left(u_{3}\right)$. Then, in this case, also $\{x, y\}$ is a half-dominating set for $\bar{G}$. Now it follows that $\gamma_{(1 / 2)} \overline{(G)}=2$.

Remark 8. We obtain the following bounds for $\mathscr{R}_{(1 / 2)}(G)+\mathscr{R}_{(1 / 2)} \overline{(G)}$, using Theorems 7, 8, and 12. If $1 \leq \delta(G)$ and $\gamma_{(1 / 2)}(G) \geq 3$, then

$$
\mathscr{R}_{(1 / 2)}(G)+\mathscr{R}_{(1 / 2)} \overline{(G)} \leq \begin{cases}2 \Delta+\delta, & \text { if } \gamma_{(1 / 2)}(G) \leq \Delta(G), \\ \Delta+\delta+1, & \text { if } \gamma_{(1 / 2)}(G)-1=\Delta(G), \\ \Delta+\delta, & \text { if } \Delta(G)<\gamma_{(1 / 2)}(G)-1 .\end{cases}
$$

Finally, we conclude this paper with the following remark on the bounds for the product $\mathscr{R}_{(1 / 2)}(G) \cdot \mathscr{R}_{(1 / 2)} \overline{(G)}$.
Remark 9. Let $k \geq 2$ be an integer. If $x+y=k$, where $x$ and $y$ are nonnegative integers, then the product $x y$ attains the maximum value if $x=y=(k / 2)$ when $k$ is even and $\{x, y\}=$ $\{((k-1) / 2),((k+1) / 2)\}$ when $k$ is odd. Thus,

$$
\max _{x+y=k} x y= \begin{cases}\left(\frac{k}{2}\right)^{2}, & \text { if } k \text { is even, } \\ \frac{(k-1)(k+1)}{4}, & \text { if } k \text { is odd. }\end{cases}
$$

Also, if $2 \leq k_{1} \leq k_{2}$, then $\left(\max _{x+y=k_{1}} x y\right) \leq\left(\max _{x+y=k_{2}} x y\right)$. As for any graph $\mathscr{R}_{(1 / 2)}(G)+\mathscr{R}_{(1 / 2)} \overline{(G)} \leq n-1$, where $|V(G)|=n$, we have an upper bound for the product $\mathscr{R}_{(1 / 2)}(G) \cdot \mathscr{R}_{(1 / 2)} \overline{(G)}$. In fact, $\mathscr{R}_{(1 / 2)}(G) \cdot \mathscr{R}_{(1 / 2)} \overline{(G)} \leq$ $\begin{cases}((n-1) / 2)^{2}, & \text { if } n \text { is odd, } \\ ((n(n-2)) / 4), & \text { if } n \text { is even. }\end{cases}$

There are graphs $G$ for which the product $\mathscr{R}_{(1 / 2)}(G)$. $\mathscr{R}_{(1 / 2)} \overline{(G)}$ attains this upper bound.

If $n \geq 5$ is odd and $G$ is a $((n-1) / 2)$-regular graph, then $\mathscr{R}_{(1 / 2)}(G) \cdot \mathscr{R}_{(1 / 2)} \overline{(G)}=((n-1) / 2)^{2}$ and $\mathscr{R}_{(1 / 2)}(G)=\mathscr{R}_{(1 / 2)}$ $\overline{(G)}=2$. Note that Harary graphs $H_{2 n+1, n}$ satisfy these properties. If $n \geq 3$, then, in this case, Harary graph $H_{2 n, n-1}$ can be considered as an example.

\section{Conclusion}

In this paper, we introduced the concept $\mathscr{R}_{(1 / 2)}(G)$, the reinforcement number of a graph $G$ with respect to the (1/2)-dominating number of $G$. Also, we studied various upper bounds for $\mathscr{R}_{(1 / 2)}(G)$ and exhibited that these bounds are sharp. Furthermore, we obtained Nordhaus-Gaddumtype bounds for the sum $\mathscr{R}_{(1 / 2)}(G)+\mathscr{R}_{(1 / 2)} \overline{(G)}$ and the product $\mathscr{R}_{(1 / 2)}(G) \cdot \mathscr{R}_{(1 / 2)} \overline{(G)}$.

\section{Data Availability}

No data were used to support the study.

\section{Conflicts of Interest}

The authors declare that they have no conflicts of interest.

\section{Acknowledgments}

This work was supported by the Taif University Researchers Supporting Project (TURSP-2020/246), Taif University, Taif, Saudi Arabia.

\section{References}

[1] R. Balakrishnan and K. Ranganathan, A Text Book of Graph Theory, Springer, London, UK, 2012.

[2] S. Amutha and N. Sridharan, " $\gamma_{t}$-Graph of a Graph G," Ramanujan Mathematical Society, Discrete Mathematics, vol. 7, pp. 255-262, 2006.

[3] H. Guoliang, "Italian reinforcement number in graphs," IEEE Access, vol. 7, pp. 448-456, 2019. 
[4] J. Amjadi, S. Nazari-Moghaddam, S. M. Sheikholeslami, and L. Volkmann, "Total Roman domination number of trees," Australas," Journal of Combinations, vol. 69, pp. 271-285, 2017.

[5] N. Sridharan, S. Amutha, and S. B. Rao, "Induced subgraphs of gamma graphs," Journal of Discrete Mathematical Sciences and Cryptography, vol. 5, no. 3, pp. 1-5, 2013.

[6] K. S. Prabha, S. Amutha, N. Anbazhagan, and S. S. Gomathi, "Neighborhood outer split domination in graphs," Journal of Discrete Mathematical Sciences and Cryptography, vol. 22, no. 5, pp. 787-799, 2019.

[7] V. R. Kulli and B. Janakiraman, "The Cobondage number of a graph," Disussiones Mathematical Graph Theory, vol. 16, pp. 111-117, 1996.

[8] T. W. Haynes, S. T. Hedetniemi, and P. J. Slater, Fundamental of Domination in Graphs, Marcel Dekker.Inc., London, UK, 1998.

[9] N. Sridharan, MD. Elias, and V. S. A. Subramanian, "Total reinforcement number of a graph," Discussiones Mathematicae Graph Theory, vol. 4, no. 2, pp. 197-202, 2007.

[10] H. A. Ahangar, J. Amjadi, M. Chellali, S. Nazari-Moghaddam, and S. M. Sheikholeslami, "Total Roman reinforcement in graphs," Discussiones Mathematicae Graph Theory, vol. 39, no. 4, pp. 787-803, 2019.

[11] N. Jafari Rad, "On the complexity of reinforcement in graphs," Discussiones Mathematicae Graph Theory, vol. 36, no. 4, pp. 877-887, 2016.

[12] H. Abdollahzadeh Ahangar, M. A. Henning, V. Samodivkin, and I. G. Yero, "Total Roman domination in graphs," Discussiones Mathematicae Graph Theory, vol. 10, pp. 501-517, 2016.

[13] H. Rashmanlou, G. Muhiuddin, S. Amanathulla, F. Mofidnakhaei, and M. Pal, "A study on cubic graphs with novel application," Journal of Intelligent \& Fuzzy Systems, vol. 40, no. 1, pp. 89-101, 2021.

[14] S. S. Hussain, R. J. Hussain, and G. Muhiuddin, "Neutrosophic vague line graphs," Neutrosophic Sets and Systems, vol. 36, pp. 121-130, 2020.

[15] G. Muhiuddin, M. Mohseni Takallo, Y. B. Jun, and R. A. Borzooei, "Cubic graphs and their application to a traffic flow problem," International Journal of Computational Intelligence Systems, vol. 13, no. 1, pp. 1265-1280, 2020.

[16] T. Pramanik, G. Muhiuddin, A. M. Alanazi, and M. Pal, "An extension of fuzzy competition graph and its uses in manufacturing industries," Mathematics, vol. 8, no. 6, p. 1008, 2020.

[17] S. Samanta, G. Muhiuddin, A. M. Alanazi, and K. Das, "Mathematical approach on representation of competitions: competition cluster hypergraphs," Mathematical Problems in Engineering, vol. 2020, 2020.

[18] A. R. Babu, " $\lambda$-domination number of a graph," Sridharan sumbitted to Alagappa University, vol. 2010, 2010.

[19] N. Sridharan, S. Amutha, and A. Ramesh Babu, "Bounds for $\lambda$ - domination number $\gamma_{\lambda}(G)$ of a graph," Journal of Computational Mathematica, vol. 1, no. 2, pp. 78-90, 2017. 